\title{
Design and evaluation of PEGylated liposomal formulation of a novel multi- kinase inhibitor for enhanced chemosensitivity and inhibition of metastatic pancreatic ductal adenocarcinoma
}

Vijay Sagar Madamsetty ${ }^{1}$ Krishnendu Pal, ${ }^{1}$ Shamit Kumar Dutta, ${ }^{1}$ Enfeng Wang, ${ }^{1}$ James R Thompson, ${ }^{2}$ Raj Kumar Banerjee, ${ }^{3}$ Thomas R. Caulfield, ${ }^{4}$ Kabir Mody, ${ }^{5}$ Yun Yen, ${ }^{6,7}$ Debabrata Mukhopadhyay, ${ }^{1,8^{*}}$ Hsu-Shan Huang ${ }^{6,7,8^{*}}$

${ }^{1}$ Department of Biochemistry and Molecular Biology, Mayo Clinic College of Medicine and Science, Jacksonville, FL,32224 USA

${ }^{2}$ SunMoon Research Partners LLC. Jacksonville, FL,32224 USA

${ }^{3}$ Department of Applied Biology, CSIR-Indian Institute of Chemical Technology, Hyderabad 500007 T.S., India; Academy of Scientific and Innovative Research (AcSIR), CSIR- Human Resource Development Centre, (CSIR-HRDC) Campus, Ghaziabad, Uttar Pradesh, 201002 , India.

${ }^{4}$ Department of Neuroscience, Mayo Clinic College of Medicine and Science, Jacksonville, FL 32224, United States.

${ }^{5}$ Division of Oncology, Department of Internal Medicine, Mayo Clinic, Jacksonville, FL,32224 United States.

${ }^{6} \mathrm{Ph} . \mathrm{D}$. Program for Cancer Molecular Biology and Drug Discovery, College of Medical Science and Technology, Taipei Medical University, Taipei 110, Taiwan, ROC 
${ }^{7}$ Graduate Institute of Cancer Biology and Drug Discovery, College of Medical Science and Technology, Taipei Medical University, Taipei 110, Taiwan, ROC

${ }^{8}$ Equal contributing senior authors

*Corresponding authors:

1. Prof. Dr. Debabrata Mukhopadhyay, Department of Biochemistry and Molecular Biology, Mayo Clinic College of Medicine and Science, USA. Tel: 1-507-2023629, e-mail: Mukhopadhyay.Debabrata@mayo.edu

2. Prof. Dr. Hsu-Shan Huang, Ph.D. Program for Cancer Molecular Biology and Drug Discovery, College of Medical Science and Technology, Taipei Medical University, Taipei, Taiwan, ROC and Graduate Institute of Cancer Biology and Drug Discovery, College of Medical Science and Technology, Taipei Medical University, Taipei 110, Taiwan, ROC Tel: +886-26638-2736 ext 1377, e-mail: huanghs99@tmu.edu.tw

\section{Methods}

\section{Molecular docking}

We performed a molecular docking analysis to investigate the binding conformation of N19 in cMET and EGFR. Kinase inhibitors can be roughly grouped into three types (I, II, and III). ${ }^{1,2}$ Type-I inhibitors target the active DFG-in conformation and compete with ATP. Type II inhibitors bind the inactive DFG-out confirmation. ${ }^{2}$ The inactive DFG-out confirmation creates an additional pocket (called back pocket) adjacent the ATP-binding site. ${ }^{2}$ Shapes of type II inhibitors are long and slender to simultaneously occupy the ATP-binding site and the back 
pocket by a head group and a tail group, respectively. The tail group is hydrophobic and forms additional interactions with the back pocket. ${ }^{2,}{ }^{3}$ N19 is more likely to be a Type-I inhibitor because its shape is not slender and lacks a hydrophobic tail group. However, cMET and EGFR structures that are currently available in the Protein Data Bank have multiple DFGconformations. For cMET and EGFR, we modeled the protein structure in with a preference for the active DFG-in conformation using a homology-modeling approach. ${ }^{4}$ The structure (PDB code $2 \mathrm{PVF}$ ) of FGFR2 with a sequence identity of $57 \%$ was selected as a structure template because it is in the active DFG-in conformation and contains an ATP analog, ACP. Additionally, docking was completed directly on well-resolved X-ray structures for cMET and EGFR using PDB codes 3CCN and 3W33 with $1.9 \AA$ and $1.7 \AA$ resolution, respectively. These structures were resolved in the presence of cofactors allowing excellent grid mapping. The residues within $10 \AA$ around the ATP analog defined the binding site. Then, N19 was docked into the binding site using XP Glide docking on Schrodinger. XP Glide uses additional precision settings and provides interaction profiles between compounds and protein residues. XP Glide uses welldocumented scoring function to determine intermolecular energies, including electrostatic, van der Waals, and hydrogen-bonding interactions. Our previous studies using XP Glide has had excellent results for drug binding and development with structure-function studies. ${ }^{5-10}$ In addition, XP Glide has been successfully applied to discover new inhibitors and binding sites for several proteins. ${ }^{11-14}$ 


\section{Supplementary Figures:}

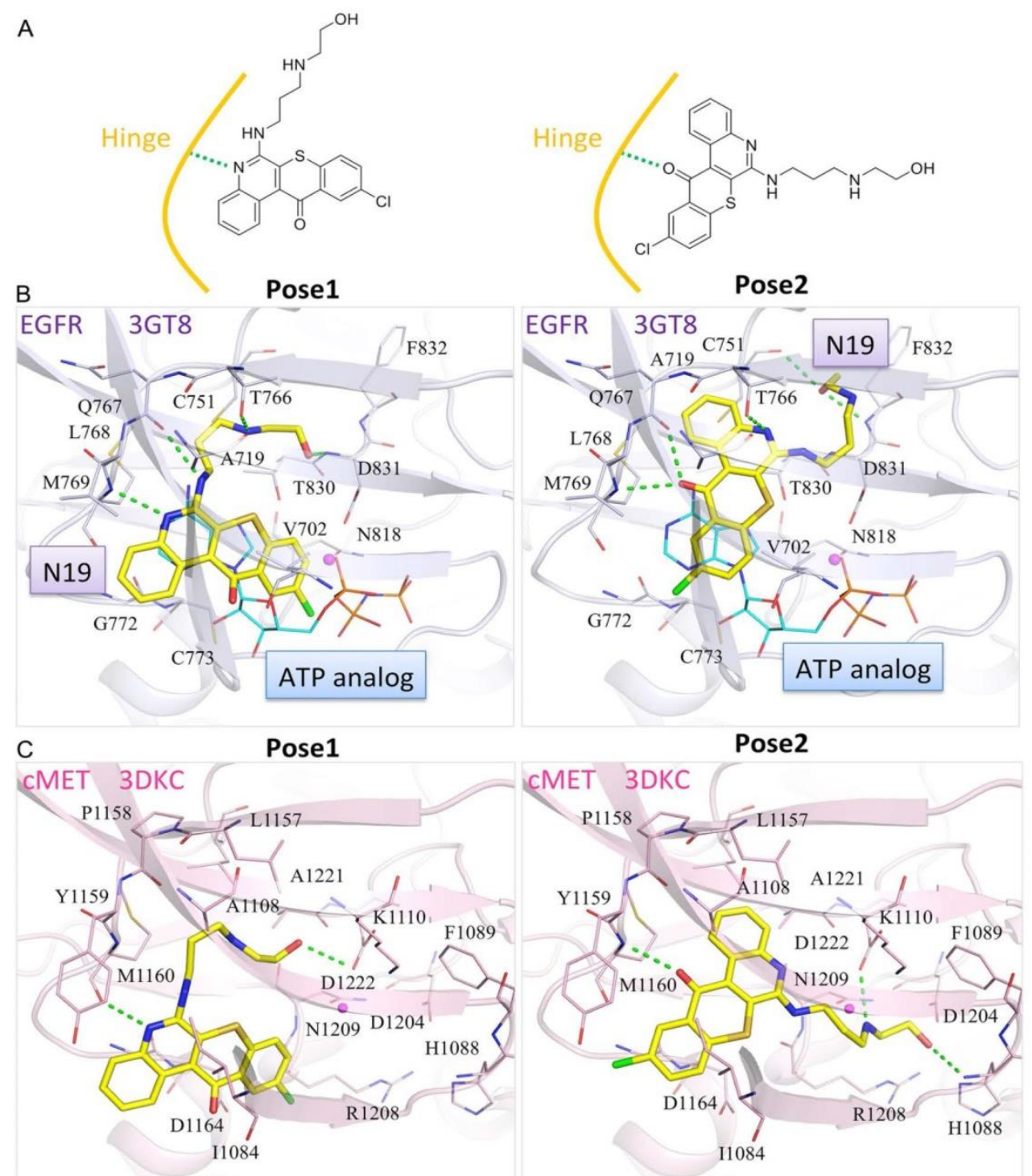

Figure S1. Binding confirmation of N19 in cMET and EGFR. A. Schematic representation of the protein binding pocket position relative to the N19 compound with pose 1 and pose 2 for orientation of the nitrogen versus ketone oxygen to the pocket hinge point. B. EGFR binding poses for N19 are given, where the ring and chain groups of N19 form 3 and 2 hydrogen bonds with EGFR residues, respectively. C. Similarly, the cMET binding poses for N19 are given, where the very similar hydrogen-bonding interactions are occurring. In both B and C panels, 
hydrogen bonds between residues and N19 are represented as light green dashes. The ring group also makes stable van der Waals interactions with the binding site, which are depicted.

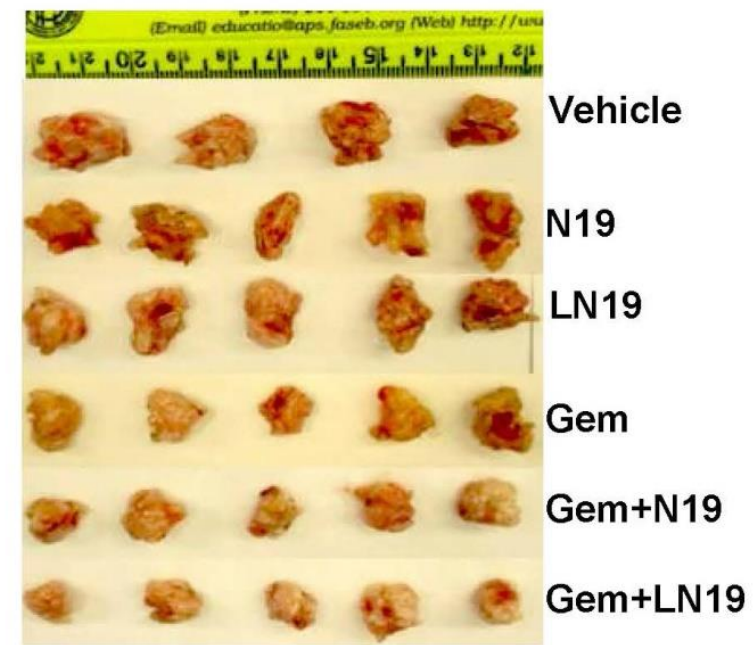

Figure S2: Representative examples of tumors treated with indicated groups.
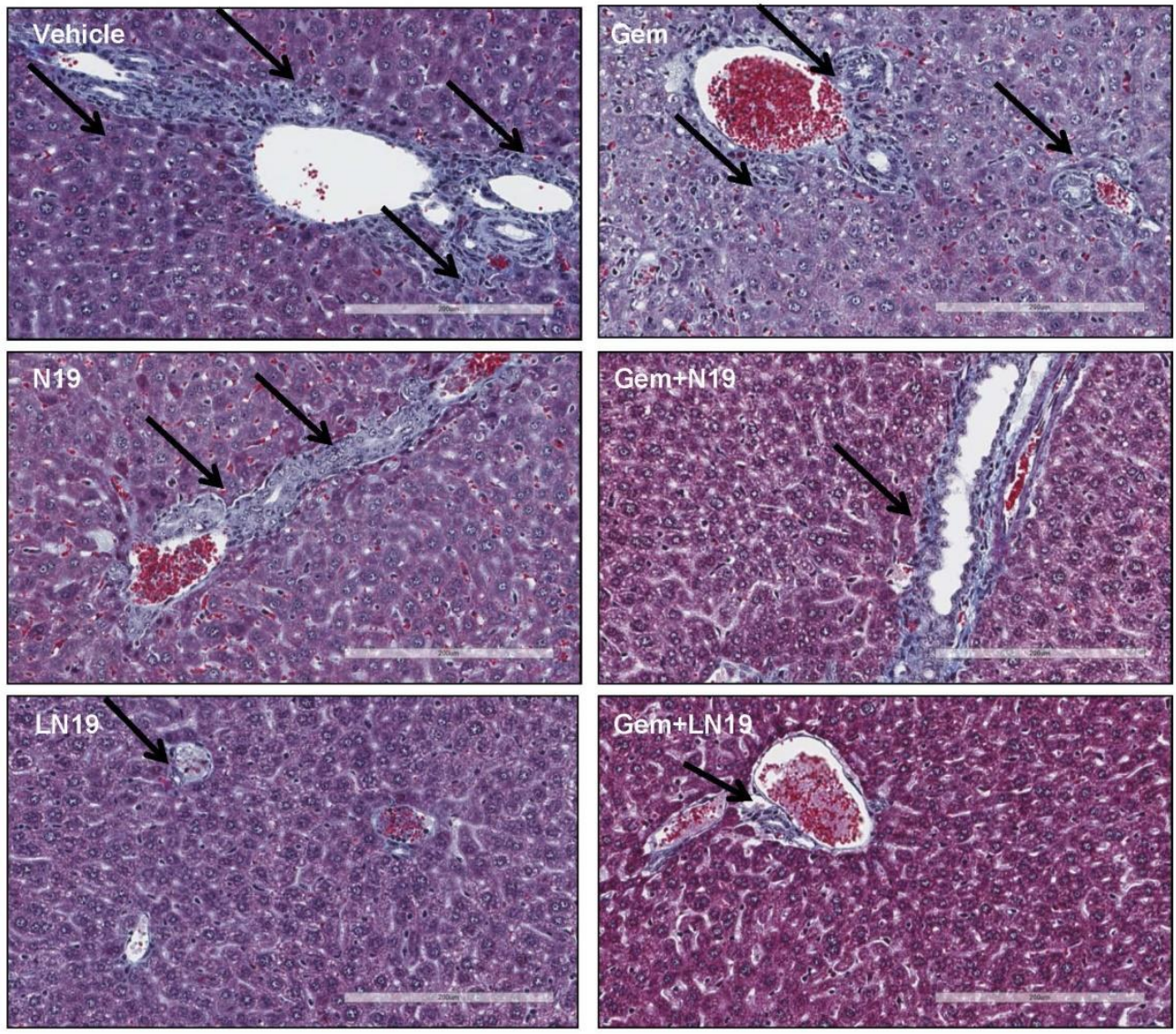
Figure S3: Immunohistochemistry of liver tissue for trichrome staining after $2 \mathrm{x} / \mathrm{wk}$ for three weeks of treatment with indicated groups. Scale bars $=200 \mu \mathrm{m}$.

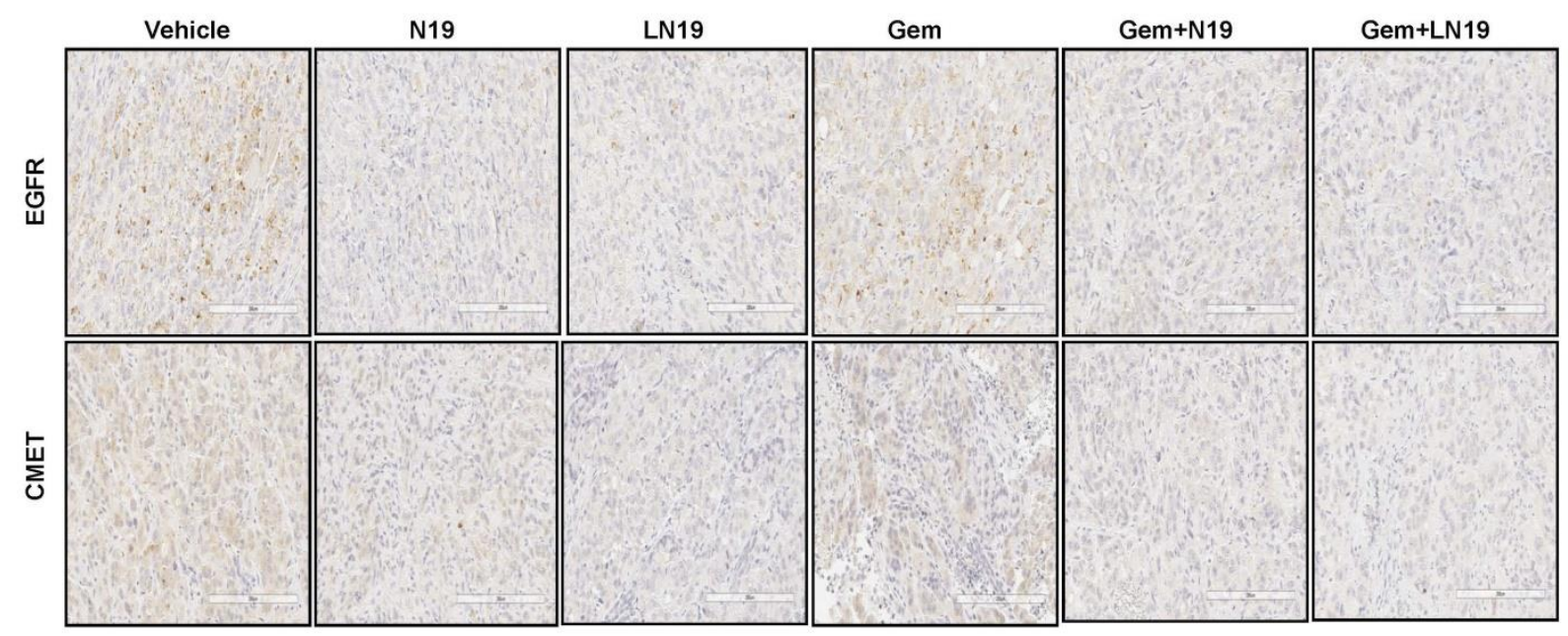

Figure S4: N19 inhibits both EGFR and cMET in PDAC tumors. IHC staining with antiEGFR (top panel) and anti-cMET (bottom panel) of tumor tissues 2x/wk for three weeks treated with indicated groups. Scale bars $=200 \mu \mathrm{m}$.

Table S1: Primers used in the study

\begin{tabular}{|l|c|}
\hline cMET-F & 5'CATGCCGACAAGTGCAGTA3' \\
\hline cMET-R & 5'TCTTGCCATCATTGTCCAAC3' \\
\hline EGFR -F & 5'GCGTCTCTTGCCGGAATGT3' \\
\hline EGFR-R & 5'GGCTCACCCTCCAGAAGGTT3' \\
\hline
\end{tabular}

Table S2. Docking results for N19 with cMET and EGFR in multiple poses.

\begin{tabular}{|l|l|l|l|}
\hline Compounds & Protein & Docking Score (kcal/mol) & Pose Rank \\
\hline N19 (pose1) & cMET & -5.87 & 3 \\
\hline N19 (pose2) & cMET & -5.3 & 4 \\
\hline Decoy Cmpd & cMET & -4.0 & 5 \\
\hline N19 (pose1) & EGFR & $-7.03^{* *}$ & 1 \\
\hline
\end{tabular}




\begin{tabular}{|l|l|l|l|}
\hline N19 (pose2) & EGFR & -6.89 & 2 \\
\hline Decoy Cmpd $^{*}$ & EGFR & -3.51 & 6 \\
\hline
\end{tabular}

"Decoy compounds were randomly selected for a counter screen on the efficacy of the N19 binding affinity for the EGFR and cMET receptors, which does indicate superiority over the randomly selected decoy compound by $>30-1000 \mathrm{X}$ depending on the pairing.

***Best docked compound pose with the highest affinity.

\section{REFERENCE}

1. Garuti, L.,Roberti, M., and Bottegoni, G. (2010) Non-ATP Competitive Protein Kinase Inhibitors. Current Medicinal Chemistry 17, 2804-2821.

2. $\quad$ Davis, M. I.,Hunt, J. P.,Herrgard, S.,Ciceri, P.,Wodicka, L. M.,Pallares, G.,Hocker, M.,Treiber, D. K., and Zarrinkar, P. P. (2011) Comprehensive analysis of kinase inhibitor selectivity. Nature Biotechnology 29, 1046-U124.

3. Liu, Y., and Gray, N. S. (2006) Rational design of inhibitors that bind to inactive kinase conformations. Nature Chemical Biology 2, 358-364.

4. Arnold, K.,Bordoli, L.,Kopp, J., and Schwede, T. (2006) The SWISS-MODEL workspace: a webbased environment for protein structure homology modelling. Bioinformatics 22, 195-201.

5. Beard, H.,Cholleti, A.,Pearlman, D.,Sherman, W., and Loving, K. A. (2013) Applying PhysicsBased Scoring to Calculate Free Energies of Binding for Single Amino Acid Mutations in Protein-Protein Complexes. PLOS ONE 8, e82849.

6. Bhachoo, J., and Beuming, T., Investigating Protein-Peptide Interactions Using the Schrödinger Computational Suite. In Modeling Peptide-Protein Interactions: Methods and Protocols, SchuelerFurman, O.; London, N., Eds. Springer New York: New York, NY, 2017; pp 235-254.

7. Harder, E.,Damm, W.,Maple, J.,Wu, C.,Reboul, M.,Xiang, J. Y.,Wang, L.,Lupyan, D.,Dahlgren, M. K.,Knight, J. L., et al. (2016) OPLS3: A Force Field Providing Broad Coverage of Drug-like Small Molecules and Proteins. Journal of Chemical Theory and Computation 12, 281-296.

8. Sindhikara, D.,Spronk, S. A.,Day, T.,Borrelli, K.,Cheney, D. L., and Posy, S. L. (2017) Improving Accuracy, Diversity, and Speed with Prime Macrocycle Conformational Sampling. Journal of Chemical Information and Modeling 57, 1881-1894.

9. Steinbrecher, T.,Abel, R.,Clark, A., and Friesner, R. (2017) Free Energy Perturbation Calculations of the Thermodynamics of Protein Side-Chain Mutations. Journal of Molecular Biology 429, 923-929.

10. Tubert-Brohman, I.,Sherman, W.,Repasky, M., and Beuming, T. (2013) Improved Docking of Polypeptides with Glide. Journal of Chemical Information and Modeling 53, 1689-1699.

11. Caulfield, T., and Medina-Franco, J. L. (2011) Molecular dynamics simulations of human DNA methyltransferase 3B with selective inhibitor nanaomycin A. J Struct Biol 176, 185-91.

12. Caulfield, T. R.,Fiesel, F. C.,Moussaud-Lamodiere, E. L.,Dourado, D. F.,Flores, S. C., and Springer, W. (2014) Phosphorylation by PINK1 releases the UBL domain and initializes the conformational opening of the E3 ubiquitin ligase Parkin. PLoS Comput Biol 10, e1003935.

13. Lopez-Vallejo, F.,Caulfield, T.,Martinez-Mayorga, K.,Giulianotti, M. A.,Nefzi, A.,Houghten, R. A., and Medina-Franco, J. L. (2011) Integrating virtual screening and combinatorial chemistry for accelerated drug discovery. Comb Chem High Throughput Screen 14, 475-87.

14. von Roemeling, C. A.,Caulfield, T. R.,Marlow, L.,Bok, I.,Wen, J.,Miller, J. L.,Hughes, 
R.,Hazlehurst, L.,Pinkerton, A. B.,Radisky, D. C., et al. (2018) Accelerated bottom-up drug design platform enables the discovery of novel stearoyl-CoA desaturase 1 inhibitors for cancer therapy. Oncotarget 9, 3-20. 